\title{
LA-UR- $08-9924$
}

Approved for public release;

distribution is unlimited.

Title:

Polarization orientation dependence of the far infrared spectra of oriented single crystals of 1,3,5,-trinitro-s-triazine (RDX) using terahertz time-domain spectroscopy.

Author(s): $\quad$ V.H. Whitley, D.E. Hooks, K.J. Ramos, J.F. O'Hara, A.K. Azad, A. J. Taylor, J. Barber, R.D. Averitt

Intended for:

Analytical and Bioanalytical Chemistry Journal

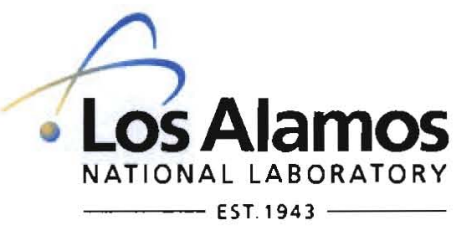

Los Alamos National Laboratory, an affirmative action/equal opportunity employer, is operated by the Los Alamos National Security, LLC for the National Nuclear Security Administration of the U.S. Department of Energy under contract DE-AC52-06NA25396. By acceptance of this article, the publisher recognizes that the U.S. Government retains a nonexclusive, royalty-free license to publish or reproduce the published form of this contribution, or to allow others to do so, for U.S. Government purposes. Los Alamos National Laboratory requests that the publisher identify this article as work performed under the auspices of the U.S. Department of Energy. Los Alamos National Laboratory strongly supports academic freedom and a researcher's right to publish; as an institution, however, the Laboratory does not endorse the viewpoint of a publication or guarantee its technical correctness. 


\title{
Polarization orientation dependence of the far infrared spectra of oriented single crystals of 1,3,5,-trinitro-s-triazine (RDX) using terahertz time-domain spectroscopy.
}

\section{V.H. Whitley, D.E.Hooks, K.J. Ramos}

DE Division, Los Alamos National Laboratory, Los Alamos, New Mexico 87545

J.F. O'Hara, A.K. Azad, A. J. Taylor

MPA-CINT, Los Alamos National Laboratory, Los Alamos, New Mexico 87545

\section{J. Barber}

Battelle,2900 Fire Rd., Suite 201, Egg Harbor Twp., NJ 08234

\section{R. D. Averitt}

Department of Physics, Boston University, Boston, MA 02215

\begin{abstract}
The far infrared spectra of (100), (010), and (001) oriented RDX single-crystals were measured as the crystal was rotated about the axis perpendicular to the polarization plane of the incident radiation. Absorption measurements were taken at temperatures of both $20 \mathrm{~K}$ and $295 \mathrm{~K}$ for all rotations using terahertz time-domain spectroscopy. A number of discrete absorptions were found ranging from 10$100 \mathrm{~cm}^{-1}(0.3-3 \mathrm{THz})$. The absorptions are highly dependent on the orientation of the terahertz polarization with respect to crystallographic axes.
\end{abstract}

\section{Introduction}


Terahertz time-domain spectroscopy (THz-TDS) shows potential as a noninvasive technique for the detection of explosives. Common energetic materials such as RDX, HMX, PETN, and TNT have unique spectral signatures in the $<100 \mathrm{~cm}^{-1}$ range.[1-15]. At visible wavelengths, many explosives look remarkably similar to common baking ingredients such as sugar or modeling clay but have unique signatures in the $\mathrm{THz}$ range. Light in the $\mathrm{THz}$ region (e.g. below $100 \mathrm{~cm}^{-1}$ ) will pass through visibly opaque materials such as clothing, luggage, paper, etc. Thus, $\mathrm{THz}$ radiation can be used to positively identify unknown substances insides opaque materials-a highly useful trait for security applications.[3, 16, 17]

While well characterized[1, 6, 9], the spectrum of RDX below $100 \mathrm{~cm}^{-1}$ is poorly understood. Measurements on powdered RDX give an absorption spectrum that is an average over many randomly oriented crystals. To complicate matters, the manufacturing process has some effect on the convoluted spectrum as well. Absorption spectra measured on RDX from different manufacturers show significant variation.[18] Assignment of absorptions to the vibrational modes of the crystal structure is difficult when using powders because the high density of absorptions make distinguishing each individual peak difficult and each absorption cannot be assigned to a given crystal orientation. Calculations on isolated RDX molecules are not able to properly account for the interactions measured in the solid phase as the majority of the absorptions seen in the solid phase are intermolecular in character[19]. Progress has been made recently into the calculated spectra of HMX and PETN in the solid phase,[20-22] but RDX is a more difficult computational problem due to the large number of molecules in the unit cell $(Z=8$ for $\alpha$-RDX[23], $Z=2$ for $\beta-H M X[24], Z=2$ for PETN[25])

Developing models that can correctly characterize the complex absorption patterns of RDX in the $<100 \mathrm{~cm}^{-1}$ region is critically important. This phonon rich region of the spectrum contains information relevant to the validation of equation of state parameters used to model the detonation process. Accurate modeling would help clarify the role these phonon modes play in shock-initiation of explosives. It has been postulated that energy is funneled from the shock into individual molecules through multiphonon up-pumping via delocalized low-frequency $(<6 \mathrm{THz})$ phonons, [2629] but little experimental data on these phonon modes exist.

In an effort to resolve and identify the vibrational modes of RDX, single crystals of $\alpha$-RDX have been studied via THz-TDS. This work is a continuation of the study published by Barber et al. in 2005.[19] Crystals cut at three orthogonal orientations were mounted and oriented via x-ray diffraction. An azimuthal rotation stage with a center hole for performing transmission spectroscopy was mounted to a cryostat, enabling spectra to be collected at $\sim 20 \mathrm{~K}$. The results of this study show terahertz absorptions not previously observed from powders or formulations.

\section{Experimental section}


For these measurements, 5 different RDX samples were used-two (100) oriented samples, two (010) oriented samples, and one (001) oriented sample. The samples were cut from large single crystals nominally $5 \mathrm{~cm}$ on a side. UK manufactured RDX was dried, purified by Soxhlet extraction in acetone and recrystallized in acetone prior to use. The crystals were grown by slow evaporation of acetone at $32^{\circ} \mathrm{C}$, in a $1000 \mathrm{~mL}$, spoutless beaker covered by a Parafilm-coated watch glass. The bottom third of the beaker was immersed in a heating bath and the top was open to air at room temperature. This method allowed for convective mixing and the reflux continuously washed the beaker sides preventing parasitic growth. Seeds were grown from acetone by a similar procedure.

Contact goniometry was used to locate planes in RDX by redundant reference to crystal facets. The samples were cut using a low speed diamond impregnated wire saw using a solution of Alconox in deionized water as a lubricant. Samples were measured in an as-cut configuration; no further polishing was performed. RDX crystals are extremely fragile, limiting the minimum sample thickness that can be cut without fracture to approximately $1 \mathrm{~mm}$. The (001) and (010) oriented samples were nominally $1 \mathrm{~mm}$ thick, the (100) sample was $1.5 \mathrm{~mm}$ thick. All samples were approximately 1 $\mathrm{cm} \times 1 \mathrm{~cm}$ wide. The orientations of the crystallographic axis and the orientation of the in-plane axes were verified by transmission Laue X-ray diffraction. Typically, the uncertainty in axis orientation is within one-half degree of the reported face. Plane indices are given with respect to the Pbca space group of RDX[23]. The structure of the RDX crystal cut along the (100), (010), and (001) faces is shown in Figure 1.

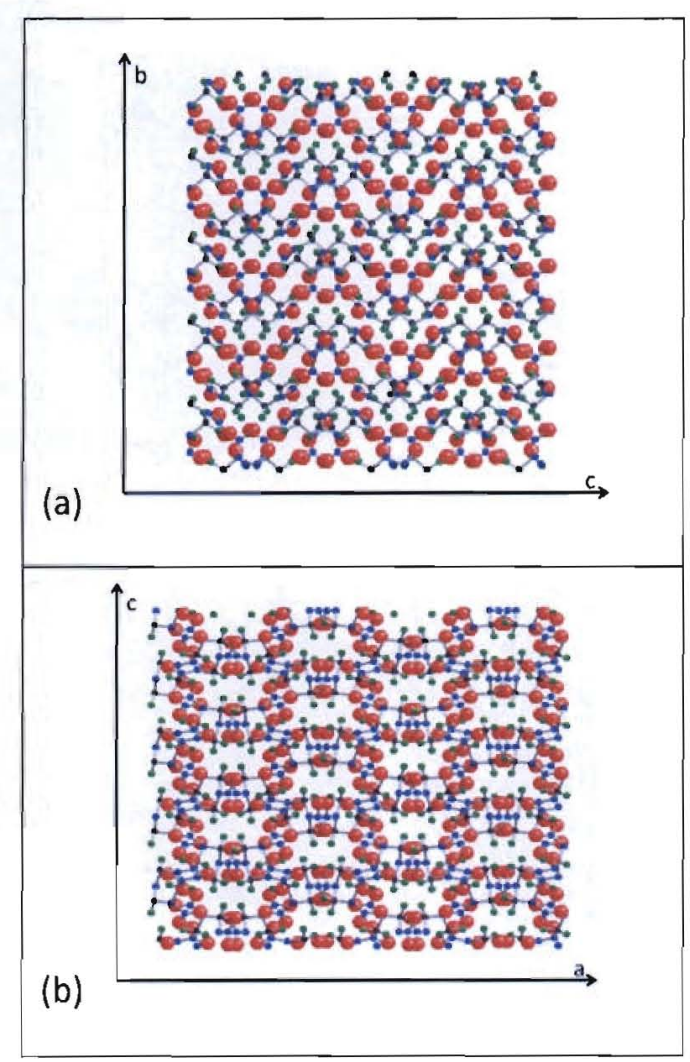




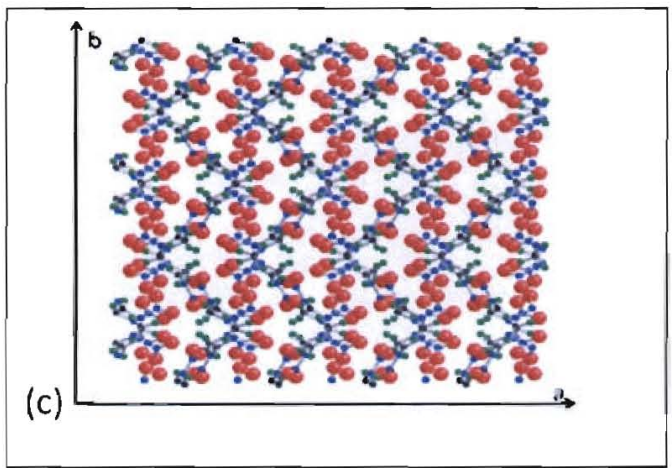

Figure 1: Representation of RDX along the primary directions(a) 100

(b) 010 (c) 001 . Carbon is shown in black, nitrogen in blue, hydrogen in green and oxygen in red.

The THz-TDS system was similar to those used in previous work[30] with some modifications to accommodate the specialized helium cryostat. Photoconductive antennas backed with silicon lenses served to both generate and detect the collimated $\mathrm{THz}$ radiation, while confocal optics guided the $\mathrm{THz}$ beam through the samples. The beam was achromatically focused to a spot about $6 \mathrm{~mm}$ in diameter at the sample face. The maximum spectral resolution of the system was $1.1 \mathrm{~cm}^{-1}$.

The cryostat and sample mounting structures were specially made to allow sample rotation and cooling under vacuum. Samples were mounted on the front end of the rotating stage, essentially a copper tube through which the focused $\mathrm{THz}$ beam propagated. The tube diameter was sufficiently large to avoid interactions with the $\mathrm{THz}$ beam. A ring gear was attached to the back of the tube and engaged to a pinion gear that was magnetically coupled through the vacuum chamber to a computer-controlled stepper motor. This allowed accurate rotational stepping of the sample orientation under vacuum. The tube was thermally connected to the main cryostat cold finger by a flexible copper band. The thermal link was sufficient to reduce the sample temperature to $18 \mathrm{~K}$ without interfering with sample rotation. Sample temperatures were monitored by a silicon sensor attached adjacent to the sample.

Initial mounting of the samples into the cryostat and orientation of the rotation stage introduced an angular inaccuracy within approximately $+/-3^{\circ}$. Samples were cooled in a helium cryostat at 0.025 $\mathrm{K} / \mathrm{s}$ to avoid cracking. Even at these rates, one each of the (010) and (100) samples cracked. Comparisons of the absorptions of the cracked and uncracked samples show that the cracks produced no significant variations in the data. Over the full 180 degree rotation, the sample temperature ranged from $18-23 \mathrm{~K}$ and had a maximum variation of $0.5 \mathrm{~K}$ during any one scan. The variation in temperature was caused by the varying thermal link of the rotation stage as it changed position. Based on the temperature-dependent absorption measurements of RDX by Melinger[15], Barber[19], and Burnett[31], the shape and position of the spectra are expected to remain unchanged over this temperature variation. 


\section{Analysis}

The time-domain data were acquired for each sample orientation over a $180^{\circ}$ rotation around the primary crystallographic axis using angle increments of $9^{\circ}$ at $22 \mathrm{~K}$ and $22.5^{\circ}$ at $295 \mathrm{~K}$. Reference data sets with the sample moved out of the path of the beam were taken at the beginning and end of each sample data set. The ratio of the Fourier transformed THz-TDS sample data to a reference data set yielded the complex transmission function. Figure 2 shows the results of a typical terahertz reference amplitude. Also shown are the transmission amplitude through $\operatorname{RDX}(010)$ with the polarization oriented along the $\mathrm{c}$-axis and the noise level of the detector/lock-in amplifier. The system is capable of producing measurable quantities of radiation to $150 \mathrm{~cm}^{-1}$. The combination of decreased emission amplitude above $\sim 100 \mathrm{~cm}^{-1}$ and strong sample absorption resulted in noisy, inconsistent data. None of the data above $100 \mathrm{~cm}^{-1}$ is discussed in this paper, but the data to 135 $\mathrm{cm}^{-1}$ is included in the data files in the electronic supplemental material. Strong absorption features, such as the $57 \mathrm{~cm}^{-1}$ feature shown in Figure 2, are completely absorbing. Since the minimum transmission is not measurable, the data produces an artificial maximum value of the absorption index $k$ or the power absorption coefficient $\alpha$.

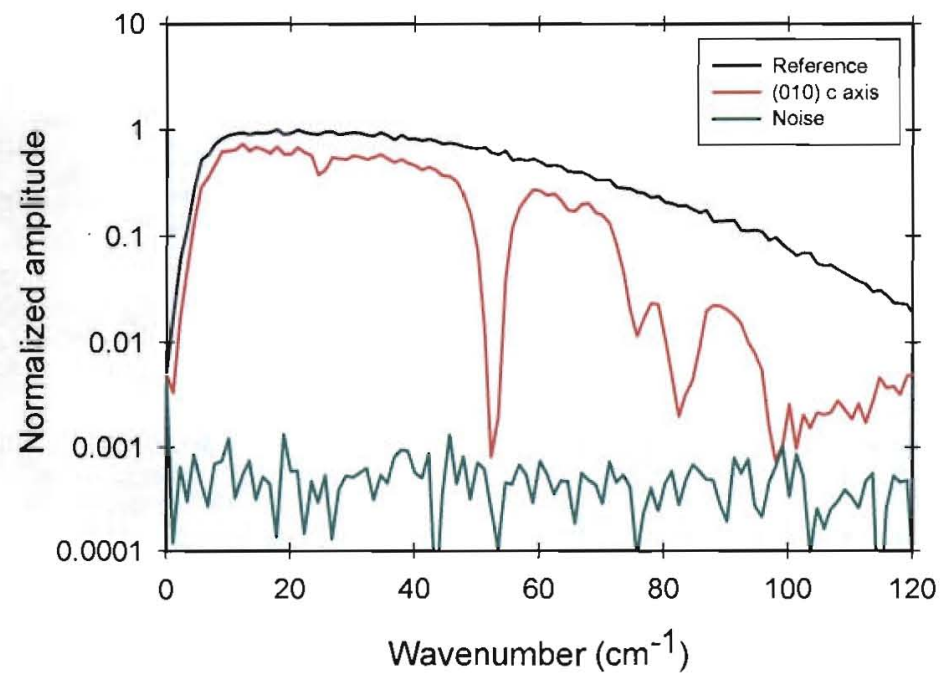

Figure 2: Electric field amplitude of the $\mathrm{THz}$ spectral emission with no sample (black), through (010) RDX with the polarization oriented along the c-axis (red), and the noise level of the detector/lock-in amplifier (green).

Since the samples are homogenous single-crystal specimen with planar faces and known thicknesses, indices of refraction and absorption values as a function of crystal orientation can be extracted. Initially, a Beer's law analysis was used to determine measured absorption coefficients (not correcting for Fresnel reflections) through the sample at each orientation. This was refined by extracting the complex index of refraction $n$ and absorption index $k$ from the THz-TDS data using the method of Duvillaret et al .[32] The similarity of the two methods provided a check that the 
fitting routines were working correctly. The fits were also verified by running the extracted results through the forward propagation equation, which matched the measured data.

\section{Results and discussions}

Figure 3-Figure 4 show the angular dependence of $k$ - and $n$-values for samples of RDX (100), (010), and (001) taken at $20 \mathrm{~K}$. The data are presented as radial contour plots with the $\mathrm{THz}$ absorption spectrum extending radially outward from the center to a maximum of $100 \mathrm{~cm}^{-1}$. Dashed circles indicate $20 \mathrm{~cm}^{-1}$ increments. The values of $n$ - or $k$ - are plotted as a function of rotation about the sample. Principle in-plane axes are indicated on the plots. Since data were taken over $180^{\circ}$ of rotation instead of a full $360^{\circ}$ rotation, the data were reproduced over the $180-360^{\circ}$ range to generate a full rotation spectrum. RDX has $\mathrm{C} 2$ symmetry so data from $0-180^{\circ}$ should be identical to $180^{\circ}-360^{\circ}$. The reader should take some caution with regions of the spectrum showing strong absorption. These absorptions are strong enough to completely block all incident $\mathrm{THz}$ radiation, so that the absorption values in these locations should be considered as a minimum instead of exact.

All three orientations of RDX in Figure 3 show a complex, feature-rich absorption structure. Significant absorption features extend from as low as $12 \mathrm{~cm}^{-1}$ to well above $100 \mathrm{~cm}^{-1}$. Below 80 $\mathrm{cm}^{-1}$, RDX has a number of rotationally-dependent absorptions strongly correlated to its crystallographic orientation with respect to the $\mathrm{THz}$ polarization, above $\sim 80 \mathrm{~cm}^{-1}$, absorptions are generally rotationally invariant.

Broad absorption features below $80 \mathrm{~cm}^{-1}$ are found along the primary crystallographic axes. The baxis has a broad absorption from $27-42 \mathrm{~cm}^{-1}$ in (001) oriented RDX and from $48-60 \mathrm{~cm}^{-1}$ in (100) oriented RDX. The a-axis has a broad absorption from $55 \mathrm{~cm}^{-1}$ to $59 \mathrm{~cm}^{-1}$ seen in both the $\mathrm{RDX}(010)$ and $\mathrm{RDX}(001)$ orientations. The $\mathrm{c}$-axis has a broad absorption from $25-40 \mathrm{~cm}^{-1}$ in the (100) orientation, although there is no corresponding c-axis absorption in the $(010)$ oriented material. In addition, there are two broad low frequency absorptions located at $45^{\circ}$ between in-plane axes. RDX(100) shows an absorption at $18 \mathrm{~cm}^{-1}$ located between the b- and c- axes and RDX(010) shows an absorption at $12 \mathrm{~cm}^{-1}$ located between the a- and b- axes.

Narrow, rotationally invariant absorptions with $\sim 1 \mathrm{~cm}^{-1}$ FWHM are found at $40 \mathrm{~cm}^{-1}$ in RDX(100), 55 $\mathrm{cm}^{-1}$ in $\mathrm{RDX}(010)$, and at $25 \mathrm{~cm}^{-1}$ in $\mathrm{RDX}(001)$. Narrow, orientation-dependent absorptions associated with the c-axis are seen in the (100) and (010) orientations at $27 \mathrm{~cm}^{-1}$. Other narrow absorptions are roughly located equally between the primary in-plane axes at $24 \mathrm{~cm}^{-1}, 48 \mathrm{~cm}^{-1}, 67$ $\mathrm{cm}^{-1}$ in RDX (001) and $40 \mathrm{~cm}^{-1}$ and $45 \mathrm{~cm}^{-1}$ in RDX(010). A summary of the in-plane absorptions found in RDX is listed in Table 1.

The absorbing states below $40 \mathrm{~cm}^{-1}$ in RDX are unique when compared to HMX[33] or PETN[34]. HMX and PETN do not show absorptions at such low frequencies. The lowest absorption for PETN is $55 \mathrm{~cm}^{-1}$ and for $\mathrm{HMX}$ is $40 \mathrm{~cm}^{-1}$. The causes of the low frequency absorption in RDX are thought to 
be related to the six extra molecules in the unit cell compared to PETN and HMX and the larger unit cell of RDX (13.192 $\AA-11.574 \AA-10.709 \AA$ for RDX[23] compared to $9.380 \AA-9.380 \AA-6.71 \AA$ for $\operatorname{PETN}(I)[25]$ and $6.54 \AA-11.05 \AA-8.7 \AA$ for HMX[24]). The absorption peaks of RDX in the $25-30 \mathrm{~cm}^{-1}$ range are particularly significant because they are responsible for the $\sim 27 \mathrm{~cm}^{-1}$ "fingerprint" peak of powdered RDX. The absorptions of single-crystal RDX below $27 \mathrm{~cm}^{-1}$, namely the broad peaks located at $18 \mathrm{~cm}^{-1}$ and $12 \mathrm{~cm}^{-1}$ in $\mathrm{RDX}(100)$ and (001)respectively, have not been previously reported. In the powdered spectrum, the $27 \mathrm{~cm}^{-1}$ absorption is a broad dominant feature that masks the $12 \mathrm{~cm}^{-1}$ and $18 \mathrm{~cm}^{-1}$ absorptions. 


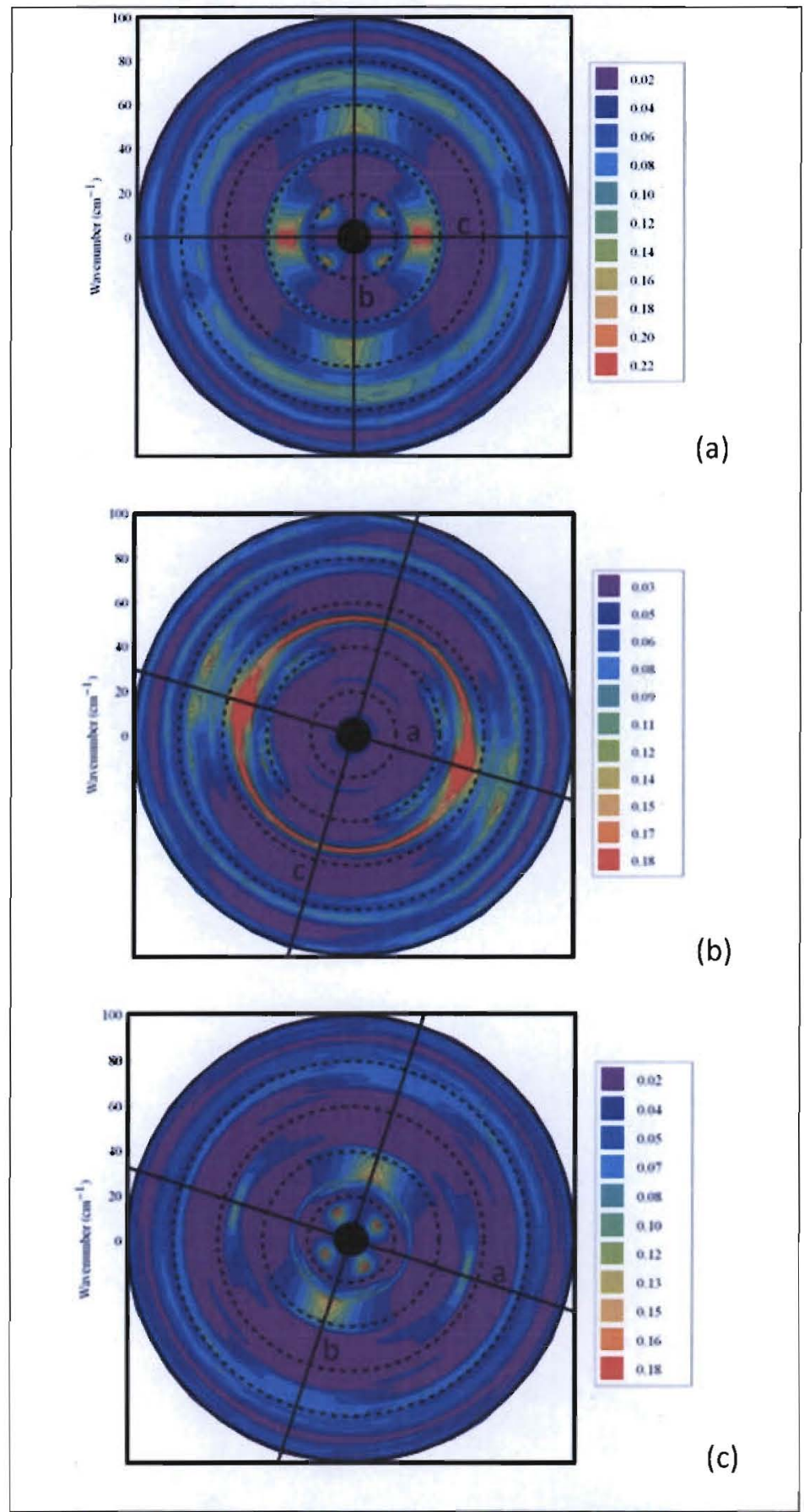

Figure 3: Comparison of the absorption index $\mathrm{K}$ of $\mathrm{RDX}(100)$, $\mathrm{RDX}(010)$ and $\mathrm{RDX}(001)$ over the 7-100 $\mathrm{cm}^{-1} \mathrm{range}$ at T=20 K. The dashed circles correspond to $20 \mathrm{~cm}^{-1}$ wavenumber intervals. Crystallographic axes are shown as the labeled solid lines.

Figure 4 shows the frequency-dependent index of refraction of single-crystal RDX as a function of orientation. The index of refraction increases from $\sim 1.6-\sim 3$ over the frequency range of $7-100 \mathrm{~cm}^{-1}$. RDX, known to be birefringent in the visible region[35], is also birefringent in the $7-100 \mathrm{~cm}^{-1}$ range. 
Symmetry points in the angular variation of the index of refraction correspond identically with the primary in-plane axes.

This birefringence can be used to find the principle in-plane axes of RDX using THz-TDS. The time delay in the onset of the THz-TDS data varies as a function of orientation. The extremes of the measured time-delay give the two in-plane axes. The minimum time delay corresponds to the axes with the smaller indices of refraction and the maximum time delay corresponds to the larger indices of refraction. For example, the minimum time delay in the pulse envelope of RDX(100) would locate the $b$-axis and the maximum delay would determine the c-axis.

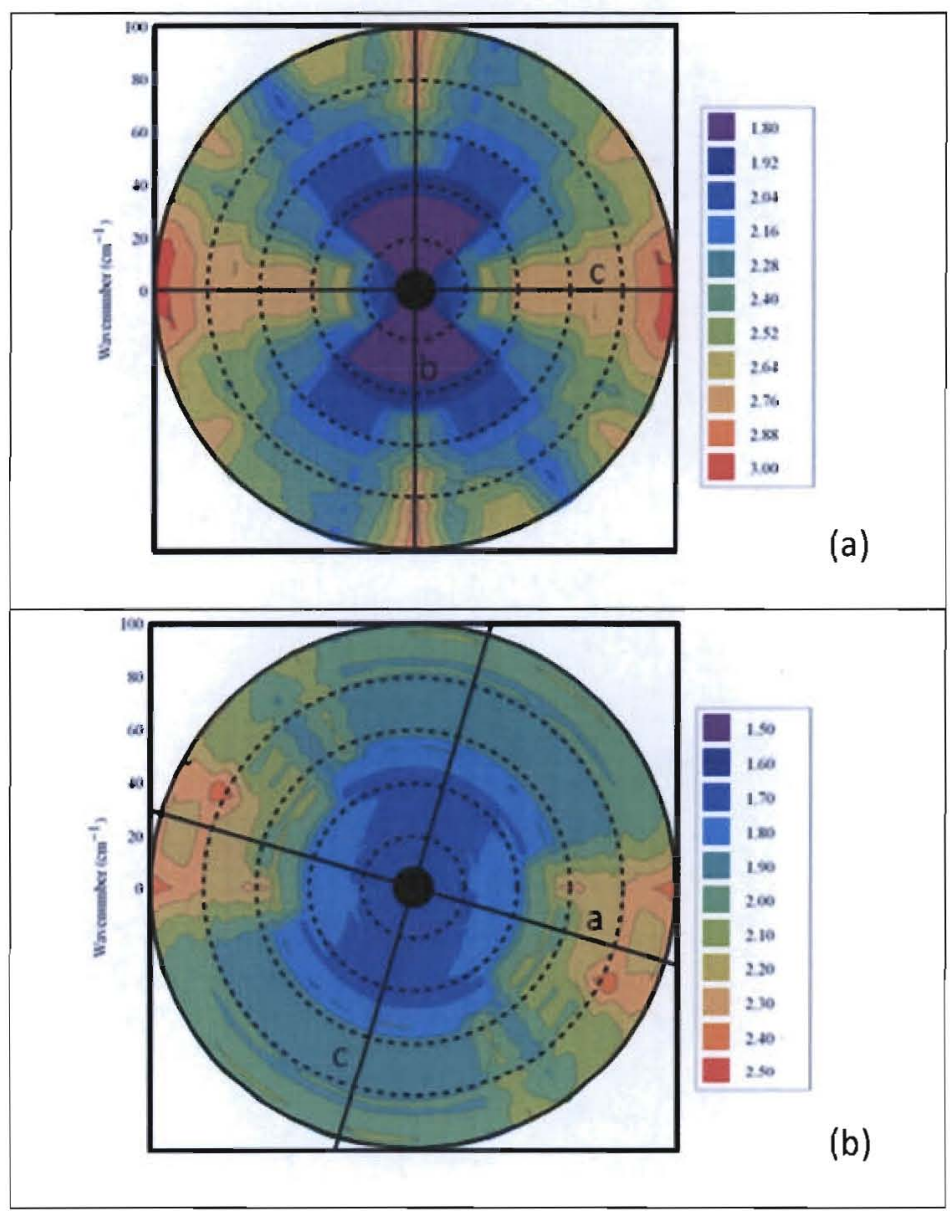




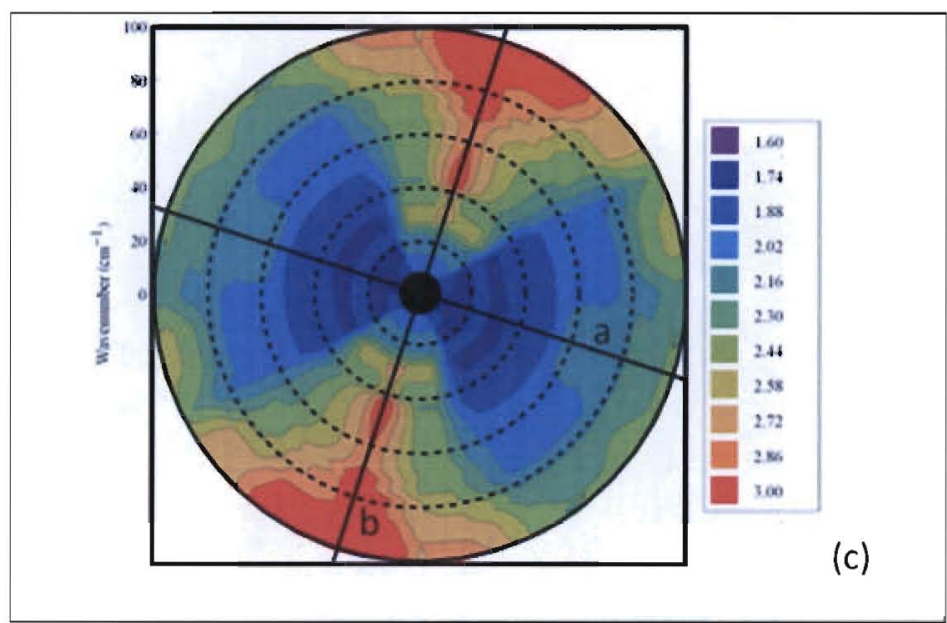

Figure 4: Comparison of the index of refraction of RDX(100), RDX(010) and RDX (001) from 7-100 $\mathrm{cm}^{-1}$ at $T=2-K_{\text {. }}$

Crystallographic axes are shown as the labeled solid lines.

Figure 5 shows a comparison of the power absorption coefficients of the three orthogonal orientations taken at $295 \mathrm{~K}$ and $20 \mathrm{~K}$. The absorption coefficients were determined through Beer's law analysis of the data and have not been corrected for Fresnel reflections. The absorption spectra of RDX at $295 \mathrm{~K}$ are very similar to the absorption spectra at $22 \mathrm{~K}$ for all orientations. Upon cooling the samples, the spectral features become sharper and shift to higher frequencies. No other major changes in the absorption are found. A summary of the frequency shifts due to temperature are included in Table 1. 

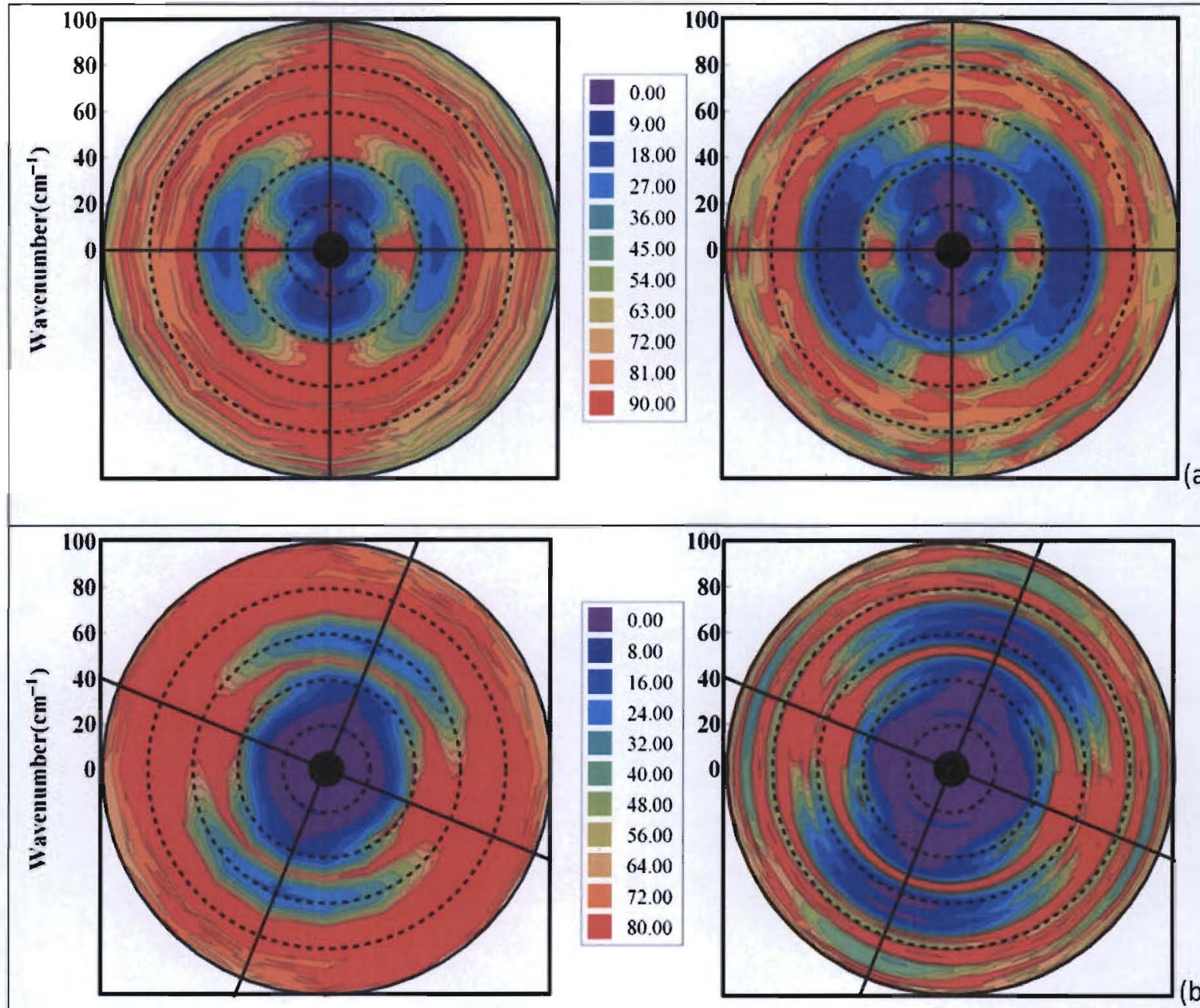

(b)
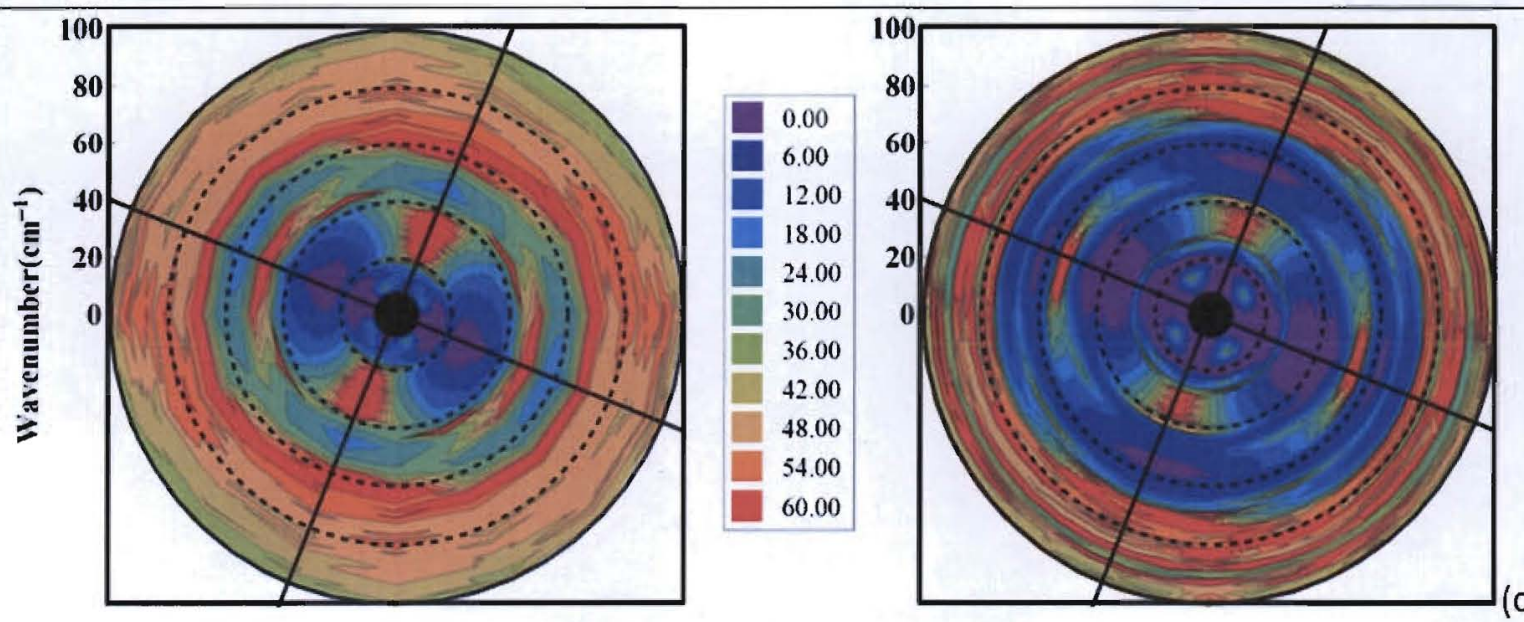

(c)

Figure 5: Comparison of the power absorption coefficient $\alpha$ for RDX(100) at 295K (left) and at 20K (right) for (a) RDX(100), (b) RDX(010) and (c) RDX(001) over the 7-100 $\mathrm{cm}^{-1}$ spectral range. The dashed circles correspond to $20 \mathrm{~cm}^{-1}$ wavenumber intervals. The contour intervals give the absorption coefficient in units of $\mathrm{cm}^{-1}$. 
This is the first work to present the rotational dependence of the absorptions of RDX single crystals, so comparison with other published work is not possible. We measured two different RDX(010) and RDX (100) samples, and performed two different measurements on the same RDX(001) sample to ensure that the data were reproducible. There were no major sample-to-sample or measurementto-measurement differences in the absorption spectra. This study does overlap with the work of Barber et $a$ l. presenting the absorption spectrum of $\operatorname{RDX}(100)$ along a single random orientation from 20-295K[19]. Comparing that spectrum with the $\operatorname{RDX}(100)$ absorptions shown above indicates that the sample used by Barber et al. was roughly oriented along the b-axis in relation to the $\mathrm{THz}$ polarization.

An averaged absorption spectrum of all the angular contributions can be compared to powder spectra if we assume that the powder spectrum is composed of an equally weighted contribution from all possible crystal orientations. To generate the equivalent of a powder absorption coefficient, we first calculate the average measured light intensity as a function of the various angles measured from all three crystal orientations:

$$
\left(\frac{I}{I_{0}}\right)=\frac{\sum_{\theta}^{n} e^{-\alpha_{\theta} t}}{n}
$$

where $\alpha_{\theta}$ is the absorption coefficient from a given angular contribution, $t$ is the thickness of the sample, and $n$ is the number of angular contributions measured. We are able to calculate this averaged intensity using the values of $\mathrm{k}$ from Figure 3 , and relating the absorption coefficient $\alpha$ to the absorption index $\mathrm{k}$ and wavelength $\lambda$ by: $\alpha=4 \pi \mathrm{k} / \lambda$. Once we have the average measured light intensity from an equal distribution of angles, then we can calculate an average absorption coefficient by solving Beer's law:

$$
\langle a\rangle=-\operatorname{Ln}\left[\left(\frac{I}{I o}\right)\right] / t
$$

Figure 6 shows the results of this analysis using the values of $\mathrm{k}$ determined from all three orientations of RDX at $22 \mathrm{~K}$. Care should be taken when viewing the averaged spectrum because the values of absorption index $\mathrm{k}$ determined for the strongest absorptions in Figure 3 are artificially truncated due to dynamic range limitations, so their contributions to the average spectrum will be artificially lowered. Nevertheless, strong absorptions are found at $27,40,51-53,75,83$, and $96 \mathrm{~cm}^{-1}$. Burnett et al. [31.] measured the spectrum of powdered RDX at $5 \mathrm{~K}$, finding absorptions at 30, 46-58, 70,80 , and $86-106 \mathrm{~cm}^{-1}$. The data presented in Figure 6 is similar to powdered RDX spectrum except for one difference: the absorption of the $27 \mathrm{~cm}^{-1}$ region in single crystals is considerably weaker than in powders. Melinger et al [15] measured high resolution absorption of ordered polycrystalline RDX films at $13 \mathrm{~K}$, finding 19 separate peaks between $0-116 \mathrm{~cm}^{-1}$. It is unclear how those peaks compare to this work or to the powdered spectrum of Burnett. If the films consisted of preferentially oriented polycrystalline RDX, as the authors suggest, we could not decipher a single orientation of the RDX films necessary to compare with the data in this paper. Perhaps the thin 
film sample was composed of several orientations with respect to the surface and the $\mathrm{THz}$ polarization.

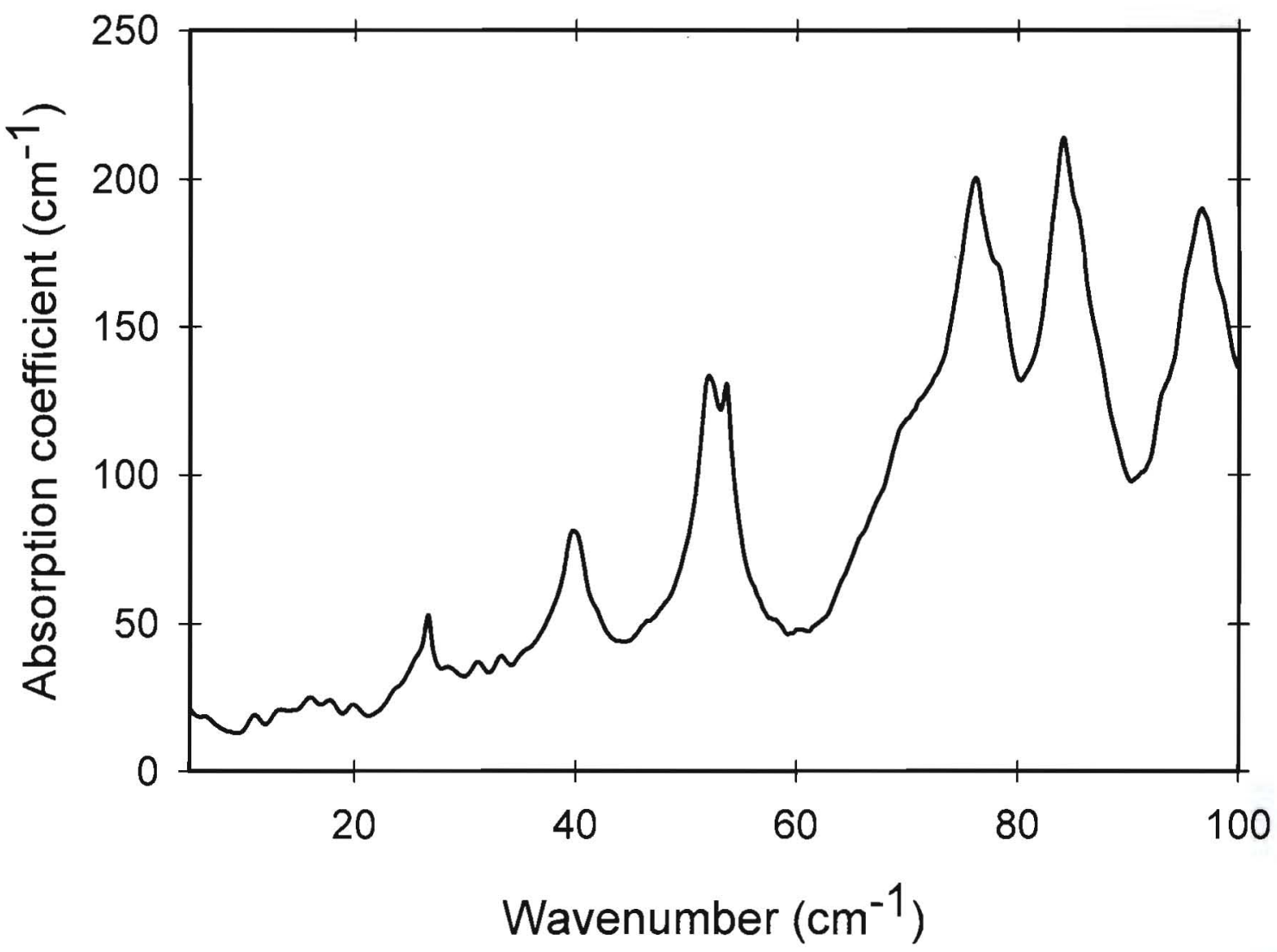

Figure 6: Average spectrum of single crystal RDX at $20 \mathrm{~K}$ composed of an equally weighted average of all the angular contributions

\section{Conclusions}

We present absorption spectra of RDX (100), (010) and (001) from 7-100 $\mathrm{cm}^{-1}$ at $295 \mathrm{~K}$ and $20 \mathrm{~K}$ as a function of orientation with respect to $\mathrm{THz}$ polarization. Results demonstrate the expected dependence of spectral features on angular orientation through alignment of the oscillating dipole to the $\mathrm{THz}$ field. Absorptions as low as $12 \mathrm{~cm}^{-1}$ have been observed, the lowest frequency absorptions reported for RDX to date. Line widths are predominantly broad ( $\left.10 \mathrm{~cm}^{-1} \mathrm{FWHM}\right)$, with some modes as narrow as $1 \mathrm{~cm}^{-1}$. It should be possible to make assignments for the spectrallyresolved absorptions by comparing these results with normal mode calculations of an $\alpha$-RDX crystal. 


\section{Acknowledgement}

Jeffrey Barber thanks Barry Smith with the Transportation Security Laboratory, Department of Homeland Security for funding support. Sample holder assemblies were designed and made by Frank Abeyta. This work supported in part by the Office of Naval Research and was performed at Los Alamos National Laboratory, operated by Los Alamos National Security, LLC, for the National Nuclear Security Administration of the U.S. Department of Energy under contract DE-AC52-06NA25396.

\section{References}

1. Kemp, M.C., et al., Security applications of terahertz technology. Proceedings of SPIE - The International Society for Optical Engineering, 2003. 5070: p. 44-52.

2. Huang, F., et al., Terahertz study of 1,3,5-trinitro-s-trizaine by time-domain and Fourier transform infrared spectroscopy. Applied Physics Letters, 2004. 85(23): p. 5535-5537.

3. Yamamoto, K., et al., Noninvasive Inspection of C-4 Explosive in Mails by Terahertz Time-Domain Spectroscopy. Japanese Journal of Applied Physics, Part II: Letters, 2004. 43(3B): p. L414-L417.

4. Chen, Y., et al. Spectroscopic characterization of explosives in the far infrared region. in Terahertz for Military and Security Applications II. 2004: Proceedings of SPIE.

5. Shen, Y.C., et al., Detection and identification of explosives using terahertz pulsed spectroscopic imaging. Applied Physics Letters, 2005. 86: p. 241116.

6. Cook, D.J., B.K. Decker, and M.G. Allen, Quantitative THz Spectroscopy of Explosive Materials, in Optical Terahertz Science and Technology 2005. 2005, Optical Society of America: Orlando, FL.

7. Hu, Y., et al., Terahertz spectroscopic investigations of explosives. Physics Letters A, 2006. 359: $p$. 728-732.

8. Zhong, H., A. Redo-Sanchez, and X.-C. Zhang, Identification and classification of chemicals using terahertz reflective spectroscopic focal-plane imaging system. Optics Express, 2006. 14(20): p. 91309141.

9. Leahy-Hoppa, M.R., et al., Wideband terahertz spectroscopy of explosives. Chemical Physics Letters, 2007. 434: p. 227-230. 
10. Fan, W.H., et al., Far-Infrared Spectroscopic Characterization of Explosives for Security Applications Using Broadband Terahertz Time-Domain Spectroscopy. Applied Spectroscopy, 2007. 61(6): p. 638-643.

11. Fitch, M.J., et al., Molecular absorption cross-section and absolute absorptivity in the $\mathrm{THz}$ frequency range for the explosives TNT, RDX, HMX, and PETN. Chemical Physics Letters, 2007. 443: $\mathrm{p}$. 284-288.

12. Chen, J., et al., Absorption coefficients of selected explosives and related compounds in the range of $0.1-2.8 \mathrm{THz}$. Optics Express, 2007. 15(19): p. 12060 - 12067.

13. Baker, C., et al., Detection of Concealed Explosives at a Distance Using Terahertz Technology. Proceedings of the IEEE, 2007. 95(8): p. 1559-1565.

14. Laman, N., et al., $7 \mathrm{GHz}$ resolution waveguide $\mathrm{THz}$ spectroscopy of explosives related solids showing new features. Optics Express, 2008. 16(6): p. 4094-4105.

15. Melinger, J.S., N. Laman, and D. Grischkowsky, The underlying terahertz vibrational spectrum of explosives solids. Applied Physics Letters, 2008. 93: p. 011102.

16. Tribe, W.R., et al. Hidden object detection: security applications of terahertz technology. in Terahertz and Gigahertz Electronics and Photonics III. 2004. San Jose, CA: Proceedings of SPIE.

17. Watters, D.G., et al., Microwave Inspection of Luggage for Contraband Materials Using Imaging and Inverse-Scattering Algorithms. Research in Nondestructive Evaluation, 1995. 7: p. 153-168.

18. Wilkinson, J., S.M. Caulder, and A. Portieri, Manufacturing process effects on the terahertz spectra of RDX, in Proceedings of the SPIE, J.O. Jensen, et al., Editors. 2008, SPIE: Orlando, FL. p. 694904.

19. Barber, J., et al., Temperature-Dependent Far-Infrared Spectra of Single Crystals of High Explosives Using Terahertz Time-Domain Spectroscopy. Journal of Physical Chemistry A, 2005. 109: p. 3501-3505.

20. Allis, D.G. and T.M. Korter, Theoretical Analysis of the Terahertz Spectrum of the High Explosive PETN. ChemPhysChem, 2006. 7(11): p. 2398-2408.

21. Allis, D.G., D.A. Prokhorova, and T.M. Korter, Solid-State Modeling of the Terahertz Spectrum of the High Explosive HMX. Journal of Physical Chemistry A, 2006. 110: p. 1951-1959.

22. Velizhanin, K.A., et al., First-principles calculations of vibrational normal modes in polyatomic materials with translational symmetry: application to PETN molecular crystal. Journal of Physical Chemistry, 2008. Submitted. 
23. Choi, C.S. and E. Prince, The Crystal Structure of Cyclotrimethylene-trinitramine. Acta Crystallographica, 1972. B28: p. 2857-2862.

24. Cady, H.H., A.C. Larson, and D.T. Cromer, The crystal structure of $\alpha-H M X$ and $a$ refinement of the structure of $\beta$-HMX. Acta Crystallographica, 1963. 16: p. 617-623.

25. Cady, H.H. and A.C. Larson, Pentaerythritol Tetranitrate II: Its crystal structure and transformation to PETN I; an algorithm for refinement of crystal structures with poor data. Acta Crystallographica, 1975. B31: p. 1864-1869.

26. Fried, L.E. and A.J. Ruggiero, Energy transfer rates in primary, secondary, and insensitive explosives. Journal of Physical Chemistry, 1994. 98: p. 9786-9791.

27. Tokmakoff, A., M.D. Fayer, and D.D. Dlott, Chemical reaction initiation and hot-spot formation in shocked energetic molecular materials. Journal of Physical Chemistry, 1993. 97: p. 1901-1913.

28. Walker, F.E., Physical Kinetics. Journal of Applied Physics, 1988. 63(11): p. 5548-5554.

29. Zerilli, F.J. and E.T. Toton, Shock-induced molecular excitation in solids. Physical Review B, 1984. 29(10): p. 5891-5902.

30. O'Hara, J.F., et al., Enhanced terahertz detection via ErAs:GaAs nanois/and superlattices. Applied Physics Letters, 2006. 88(251119).

31. Burnett, A.D., et al. Broadband terahertz time-domain and Raman spectroscopy of explosives. in Proceeding of the SPIE. 2007.

32. Duvillaret, L., F. Garet, and J.-L. Coutaz, A reliable method for extraction of material parameters in terahertz time-domain spectroscopy. IEEE Journal of Selected Topics in Quantum Electronics, 1996. 2(3): p. 739-746.

33. Whitley, V.H., et al., Orientation dependence of the far infrared spectra of oriented single crystals of HMX using terahertz time-domain spectroscopy. In Preparation, 2009.

34. Whitley, V.H., et al., Orientation dependence of the far infrared spectra of oriented single crystals of PETN using terahertz time-domain spectroscopy. In Preparation, 2009.

35. Gibbs, T.R. and A. Popolato, eds. LASL explosive property data. 1980, University of California Press, Berkley, CA. 
Table 1: Summary of absorption found in (100), (010), and (001) oriented RDX.

\begin{tabular}{|c|c|c|}
\hline Orientation & Frequency THz & $\begin{array}{c}\text { Absorption } \\
\text { coefficient }\left(\mathrm{cm}^{-1}\right)\end{array}$ \\
\hline \multirow{7}{*}{$(100)$} & $0 . \overline{48}$ & 41 \\
\hline & 0.54 & 32 \\
\hline & 0.76 & $>46$ \\
\hline & $0.82-1.15$ & 37 \\
\hline & 1.18 & $>46$ \\
\hline & $1.45-1.9$ & $>46$ \\
\hline & $2-3$ & $>46$ \\
\hline \multirow{6}{*}{ (010) } & 0.82 & 23 \\
\hline & 1 & 18 \\
\hline & $1.2-1.3$ & 23 \\
\hline & $1.5-1.67$ & $>35$ \\
\hline & $1.45-3$ & $>35$ \\
\hline & $2 . \overline{1}$ & $>3 \overline{5}$ \\
\hline \multirow{7}{*}{ (001) } & 0.36 & 18 \\
\hline & 0.72 & 35 \\
\hline & 0.79 & 35 \\
\hline & $0.85-1.2$ & $>4 \overline{6}$ \\
\hline & 1.4 & 18 \\
\hline & $1.5-1.67$ & $>46$ \\
\hline & $2.1-3$ & $>46$ \\
\hline
\end{tabular}

